\title{
Actualités en antibiothérapie - Les nouvelles molécules antibiotiques
}

\section{Latest developments in antibiotherapy - New antibiotic molecules}

\author{
O. Leroy \\ (C) SRLF et Springer-Verlag France 2011
}

Les cliniciens doivent faire face à des infections liées à des bactéries de plus en plus résistantes aux antibiotiques. Parmi les cocci à Gram positif sont apparues de nombreuses souches de Staphyloccocus aureus résistants à la méticilline, de Streptococcus pneumoniae de sensibilité diminuée, voire résistants aux pénicillines et d'entérocoques résistants aux glycopeptides. Par ailleurs, un accroissement progressif des CMI des glycopeptides à l'encontre des souches de S. aureus résistants à la méticilline peut laisser craindre une moindre efficacité clinique de ces antibiotiques. Au sein des bacilles à Gram négatif, on assiste à l'émergence des nombreuses souches d'entérobactéries productrices de bêtalactamases à spectre étendu et de souches multirésistantes de Pseudomonas aeruginosa et d'Acinetobacter baumanii. Pour faire face à cette plus fréquente ou plus grande résistante des bactéries, les nouvelles molécules antibiotiques sont malheureusement peu nombreuses.

Les nouveaux antibiotiques efficaces à l'encontre des cocci à Gram positif sont le linézolide, premier représentant d'une nouvelle classe d'antibiotiques, les oxazolidinones, et la daptomycine, premier représentant de la classe des lipopeptides cycliques. Le spectre d'activité de ces deux antibiotiques inclut la plupart des bactéries à Gram positif. En France, les indications du linézolide sont le traitement des pneumonies communautaires ou nosocomiales et celui des infections compliquées de la peau et des tissus mous. Celles de la daptomycine sont le traitement des infections compliquées de la peau et des tissus mous, des endocardites du cœur droit et des bactériémies à $S$. aureus.

Les nouvelles molécules développées face à l'émergence des bacilles à Gram négatif multirésistants appartiennent à la famille des carbapénèmes. Il s'agit de l'ertapénème et du doripénème. L'ertapénème a une excellente activité in vitro sur la plupart des entérobactéries, y compris celles produisant des bêtalactamases à spectre élargi. Toutefois, à l'inverse de l'imipénème ou du méropénème, il n'est pas actif sur $P$. aeruginosa et Acinetobacter spp. En France, ses indications sont le traitement des infections intra-abdominales compliquées, des pneumonies communautaires, des infections gynécologiques aiguës et des infections de la peau et des tissus mous du pied diabétique. Le doripénème a un spectre voisin de l'imipénème ou du méropénème avec, de plus, une activité sur certaines souches de $P$. aeruginosa résistantes à ces carbapénèmes. En France, ses indications correspondent au traitement des infections intra-abdominales ou urinaires compliquées et des pneumonies nosocomiales, y compris celles acquises sous ventilation mécanique.

La tigécycline est une molécule dont le spectre inclut à la fois des bactéries à Gram positif, y compris les souches résistantes (S. aureus résistant à la méticilline, $S$. pneumoniae de sensibilité diminuée ou résistant aux pénicillines et entérocoque résistant aux glycopeptides) et des bactéries à Gram négatif telles que les entérobactéries, même celles productrices de bêtalactamases à spectre étendu, et certains bacilles non fermentaires comme A. baumanii et $S$. maltophilia. C'est la première glycylcycline. En France, elle est indiquée dans le traitement des infections compliquées de la peau et des parties molles ou intra-abdominales.

Un certain nombre d'autres molécules sont actuellement en cours de développement ou d'essais cliniques. Il s'agit de céphalosporines efficaces à l'encontre des $S$. aureus résistants à la méticilline (ceftobiprole et ceftaroline), de dérivés de glycopeptides nommés lipoglycopeptides (dalbavancine, télavancine et oritavancine) ou de quinolones (garénoxacine).

Conflit d'intérêt : l'auteur déclare avoir perçu des honoraires d'orateur par les laboratoires MSD, Pfizer, Novartis, Wyeth et Aventis, ainsi que des honoraires d'investigateur par les laboratoires MSD et Aventis.

\section{O. Leroy $(\bowtie)$}

Service de réanimation et de maladies infectieuses, centre hospitalier Chatiliez, F-59200 Tourcoing, France

e-mail : oleroy@ch-tourcoing.fr 